\section{FIXED TEETH IN A DAY}

The All-on-4 treatment concept from Nobel Biocare is a lifechanging and viable option for edentulous patients who do not wish to wear removable dentures. The solution can be implemented quickly and, in certain cases, a patient can receive a secure fixed bridge prosthesis within a day.

The innovative treatment concept has been perfected by Nobel Biocare, in terms of speed and use of available bone, enabling practitioners to help a larger number of patients, who were previously not considered viable for implant surgery, or who were wary of the cost and treatment time.

The All-on-4 treatment concept is compatible with Nobel Guide for precision diagnosis and highly accurate placement of implants. The concept involves the placement of two straight anterior implants and two posterior implants. The latter are positioned at an incline of $30^{\circ}$ to ensure stability and support, even for patients who have minimal available bone. An additional benefit of this method is that it avoids the sinus and nerve, removing the need for lifts or repositioning, and quickening the whole process.

The All-on-4 treatment concept is an efficient procedure with minimal cost and discomfort to the patient for a truly dramatic result.

Visit www.nobelbiocare.com.

\title{
BESPOKE, COMFORTABLE ANTI-SNORING DEVICES
}

Somnowell have developed the world's first fourth generation mandibular advancement device for snoring and sleep apnoea. The Somnowell MAA is made from cast chrome cobalt alloy, making it extremely comfortable, small and discreet. It is also impervious to bacteria.

The Somnowell MAA is a bespoke device, individually designed to meet your patient's needs. It is ultra-resilient and outperforms all acrylic plastic and thermoplastic devices. The connecting mechanism can be advanced up to a further $5 \mathrm{~mm}$ from the optimum advancement set at construction.

The Somnowell MAA has minimal infringement on tongue space and free vertical and lateral jaw movement. Chrome cobalt does not suffer from wear or tear in the oral environment, so it will look and feel the same after years of use.

The Somnowell was invented by Dr Simon Ash who specialises in sleep related breathing disorders. Dr Ash is intimately involved in the construction of each Somnowell MAA and will personally oversee each appliance's design and construction.

www.somnowell.com

\section{ONE OF THE MOST EFFECTIVE IMPLANTS AVAILABLE}

Independently judged one of the most effective implants evaluated in a study by the New York University, ADIN implants are available in the UK from Trycare. NYU measured the quality of new bone formation and bone contact of five different implant systems (ADIN, Astra, IL, Nobel and Straumann) over a six-week period. It showed that all five produced very similar results, though ADIN out-performed the competitors, which are up to three times more expensive, in various aspects.

ADIN implants are available in Tourag CloseFit and Toureg S. Both have a tapered core with spiral tapping to condense bone during insertion, promoting immediate stability. The unique tip and double lead threads scrape away bone as they tap. Because they progressively condense and reposition bone during insertion ADIN implants can be easily redirected as needed, render exceptional primary stability and optimal restorative orientation.

Toureg CloseFit implants offer a strong and solid conical-hex connection that is interchangeable with
Nobel Active. This Morse Taper connection minimises micro movements, thereby minimising undesirable crestal bone loss.

Toureg S implants are multi-functional implants featuring a special dome apex. Suitable for conventional applications this domed apex also lifts bone grafts without damaging the surrounding bone, making them ideal for lifting the sinus floor.

For further information ask your local Trycare Representative, call Trycare on 01274885544 or visit www. adin.co.uk.

\section{THE SMALLEST HEAD ON THE MARKET}

The iChiropro by Bien-Air is a revolutionary physiodispenser controlled from an iPad. Simple to use, the intuitive, ergonomic application ensures significant time savings during operations. Available free from the Apple App Store, the iChiropro application has numerous customisation options.

The multi-user iChiropro incorporates the complete sequences of the leading implant manufacturers with their predefined settings. It also offers the option of saving and exporting operation data, in the form of graphics and tables, attached to the patient file built into the control interface. It's the only device which allows users to save, export and print all operation parameters, whilst documenting these with the patient data and the implants used.

Its CA 20:1 L Micro-Series contra-angle handpiece is equipped with an internal irrigation system for greater comfort with one of the smallest heads of any implant handpiece on the market. Fit- ted with an exclusive double optical glass rod system ensures uniform lighting of the operative field even at very low speeds. A Kirschner Meyer version of the CA20:1 is also available.

Claim your FREE iPad 2 with every purchase of a iChiropro complete with CA20:1 L Contraangle.

For more details or a demonstration call 01293550200 or email ba-uk@bienair.com. 